\title{
THREE PARTICULAR SYSTEMS OF LINES ON A SURFACE*
}

BY

\section{LUTHER PFAHLER EISENHART}

\section{INTRODUCTION}

Cufarelli has established the following theorem : $\dagger$

Given two quadratic differential forms

$$
\begin{aligned}
& a_{1} d u^{2}+2 a_{2} d u d v+a_{3} d v^{2}, \\
& b_{1} d u^{2}+2 b_{2} d u d v+b_{3} d v^{2},
\end{aligned}
$$

of which the first is definite, that is, $a_{1} a_{3}-a_{2}^{2}>0$; there exists a unique real transformation of variables

$$
u^{\prime}=\phi(u, v), \quad v^{\prime}=\psi(u, v),
$$

which changes the above forms into

with the relations

$$
\begin{aligned}
& a_{1}^{\prime} d{u^{\prime}}^{2}+2 a_{2}^{\prime} d u^{\prime} d v^{\prime}+a_{3}^{\prime} d v^{\prime 2}, \\
& b_{1}^{\prime} d u^{\prime 2}+2 b_{2}^{\prime} d u^{\prime} d v^{\prime}+b_{3}^{\prime} d v^{\prime 2},
\end{aligned}
$$

$$
\frac{a_{1}^{\prime}}{b_{1}^{\prime}}=\frac{a_{3}^{\prime}}{b_{3}^{\prime}}, \quad a_{2}^{\prime}=0 .
$$

In order to find the transformation $(b)$ which effects this change, one takes the Jacobian of the forms $(a)$ and then in turn forms the Jacobian of this quadratic form and the first of $(a)$. The resulting quadratic differential form has real linear factors; when the latter are multiplied by integrating factors and the products equated to $d u^{\prime}$ and $d v^{\prime}$ respectively, the required transformation is given by quadratures.

We apply this theorem to the two fundamental forms of a surface $S$ written as follows:

$$
\begin{aligned}
d s^{2} & =E d u^{2}+2 F d u d v+G d v^{2} \\
\phi^{2} & =D d u^{2}+2 D^{\prime} d u d v+D^{\prime \prime} d v^{2}
\end{aligned}
$$

and to the form

$$
d \sigma^{2}=e d u^{2}+2 f d u d v+g d v^{2},
$$

* Presented to the Society February 27, 1904. Received for publication January 2, 1904.

† Le Congruenze, Annali di Matematica, ser. III, vol. 2, 1899. 
which represents the square of the linear element of the spherical representation of $S$. Between these three quadratic forms the following linear relation holds: *

$$
K d s^{2}+H \phi^{2}+d \sigma^{2}=0,
$$

where $K$ and $H$ denote the total and mean curvatures respectively of the surface. The forms (I) and (III) are definite for all real surfaces, whereas the form (II) is definite only for surfaces of positive total curvature. In consequence of the above relation there are only three independent ways in which the transformation $(b)$ can be applied to the above forms. When the surface is referred to any one of the three parametric systems so defined, the fundamental quantities for the surface satisfy the relations:

and one of three

$$
\frac{E}{D}=\frac{G}{D^{\prime \prime}}, \quad \frac{E}{e}=\frac{G}{g},
$$

$$
1^{\circ}, F=0 ; \quad 2^{\circ}, f=0 ; \quad 3^{\circ}, D^{\prime}=0 .
$$

The parameters corresponding to the case $3^{\circ}$ are real only for surfaces of positive constant curvature.

We proceed in this paper to the consideration of the three particular systems of lines upon a surface which are characterized by the above relations when the lines are parametric. We consider for the lines of each system a length defined as follows: Let the surface be referred to one of the systems and at the points $(u, v),(u+d u, v),(u, v+d v)$ draw normals to the surface. Denote by $r_{u}$ and $r_{v}$ the lengths measured on the first from the point $(u, v)$ to the points where this line is met by the lines of shortest distance to the second and the third lines respectively. We shall refer to this length as the distance $r$. It is found that the function $r$ and the radii of normal and geodesic curvature of the directions of the lines of all three systems are simple functions of the radii of principal curvature of the surfaces. These forms are tabulated in $\S 3$.

In $\S 1$ the system of lines satisfying conditions $(c)$ and $1^{\circ}$ is studied. Their directions form angles of $45^{\circ}$ with the tangents to the lines of curvature; on this account we call them the mean orthogonal lines. When $S$ is an isothermic surface, and only in this case, the mean orthogonal lines form an isothermal system.

The system corresponding to the conditions $(c)$ and $2^{\circ}$ is treated in $\$ 2$ and it is found that the tangents to these curves are conjugate to the directions of the mean orthogonal lines. In the case of minimal surfaces and the sphere these curves coincide with the mean orthogonal lines. The spherical representation of these lines gives an orthogonal system and it is shown that every orthogonal system on the sphere is the image of these lines on a number of sur-

* Braxchi, Lezioni, p. 116. 
faces. The determination of these surfaces, when the system on the sphere is given, requires the integration of a partial differential equation of the second order and quadratures. When the system on the sphere is isothermal, the lines of curvature of the corresponding surfaces have an isothermal spherical representation.

In $\S 3$ it is shown that the angle between directions in the system characterized by $(c)$ and $3^{\circ}$ is bisected by the directions of the lines of curvature and that no other conjugate system has this property. The existence of such a system was discovered by HOPPE* in seeking to find the conjugate system on a surface of positive curvature whose included angle is a minimum. Later PuCCI, $\dagger$ in considering the same problem, called these the characteristic lines and the included angle the characteristic angle. We shall make use of these terms. We find that this angle is constant only for those Weingarten surfaces for which the ratio of the principal curvature is constant. Recalling that Weingarten surfaces are characterized by the property that asymptotic lines on the sheets of the evolute correspond, we inquire whether there are any surfaces which have a similar property for characteristic lines and find that in every case the sheets are of negative curvature and consequently the characteristic lines are imaginary.

In $\S 4$ we consider the spherical representation of characteristic lines and find the conditions which a system of lines on the sphere must satisfy in order that they may be the image of the characteristic lines. When this condition is satisfied and the coördinates of a point on the sphere are known, the further determination of the surface reduces to quadratures. As in the case of asymptotic lines the surface is unique for each system on the sphere. The expressions for the cartesian coördinates of such a surface are reduced to forms similar to the Lelieuvre $\ddagger$ formulæ for surfaces whose parametric lines are asymptotic.

The paper closes with a proof of the impossibility of the successive application of the theorem of CrFARELLI for the determination of new systems of lines.

* Ueber das Minimum des Winkels, etc., Grünert's A rchiv, vol. 69, pp. 19-30.

$\dagger$ Dell'angolo caratteristico e delle linee caratterische di una superficie, Rom. A cc. L. Rend. (4), V (1889), pp. 501-7. In a paper entitled Di alcune proprieta delle linee caratterische (Rom. A cc. L. Rend. (4), V (1889), pp. 881-5) Reini showed that if normals be drawn to a surface at the points of a geodesic circle of radius $d s$ and center $(u, v)$, the distance between the normal at the latter point and those at the points of the circle in the direction of the characteristic lines is the maximum. RAFFY also has studied these lines in an article entitled Sur le reseau diagonal conjugue (Bull. de la Soc. Math. de France, vol. 30 (1902), p. 226). He set out to study the conjugate directions on a surface corresponding to equal conjugate diameters of the Dupin indicatrix of the surface. The lines satisfying this condition are the characteristic lines, as is evident from the fact that the normal radii are equal in these directions, but RAFFY does not call attention to this fact. From his definition he finds equation (24) for the definition of the directions of these lines and then he considers the forms which this equation takes when certain lines are parametric.

$\ddagger$ BraNeHI, Lezioni, p. 130. 


\section{§1. Mean orthogonal lines.}

We consider first that system of lines upon every surface which are such that when they are parametric the fundamental quantities satisfy the conditions

$$
\frac{E}{D}=\frac{G}{D^{\prime \prime}}, \quad \frac{E}{e}=\frac{G}{g}, \quad F=0 .
$$

From the general theorem it follows that, when the surface is referred to any system of lines whatever, the directions of this special system are given by the equation

$$
\begin{array}{r}
{\left[E\left(G D-E D^{\prime \prime}\right)-2 F\left(F D-E D^{\prime}\right)\right] d u^{2}+\left[4 E G D^{\prime}-2 F\left(E D^{\prime \prime}+G D\right)\right] d u d v} \\
+\left[2 F\left(G D^{\prime}-F D^{\prime \prime}\right)-G\left(G D-E D^{\prime \prime}\right)\right] d v^{2}=0 .
\end{array}
$$

When in particular the parametric lines are the lines of curvature of the surface, the above equation reduces to

$$
E_{1} d u_{1}^{2}-G_{1} d v_{1}^{2}=0
$$

where the subscript denotes functions corresponding to the lines of curvature. If we denote by $\theta$ the angle which these lines make with the direction of the line of curvature $v=$ const., we have

$$
\tan \theta= \pm 1
$$

that is, these curves bisect the angles between the lines of curvature. Hence we say that they form the mean orthogonal system on the surface.

From the general form of the equation of the directions of the lines of curvature *

$$
\left(E D^{\prime}-F D\right) d u^{2}+\left(E D^{\prime \prime}-G D\right) d u d v+\left(F D^{\prime \prime}-G D^{\prime}\right) d v^{2}=0,
$$

it follows that when the surface is referred to its mean orthogonal system the lines of curvature are given by

$$
E d u^{2}-G d v^{2}=0 .
$$

Denoting by $\rho_{u}$ and $\rho_{v}$ the radii of normal curvature of the surface in the directions of the mean orthogonal lines $v=$ const., $u=$ const., respectively, we have from (1)

By means of Euler's equation

$$
\rho_{u}=\rho_{v}
$$

$$
\frac{1}{\rho}=\left(\frac{\cos ^{2} \omega}{\rho_{1}}+\frac{\sin ^{2} \omega}{\rho_{2}}\right)
$$

\footnotetext{
*Bianchi, l. c., p. 99.
} 
where $\rho_{1}$ and $\rho_{2}$ are the principal radii and $\omega$ is the angle made by the section of radius $\rho$ with the direction corresponding to $\rho_{1}$, we have

Hence :

$$
\frac{1}{\rho_{u}}=\frac{1}{\rho_{v}}=\frac{1}{2}\left(\frac{1}{\rho_{1}}+\frac{1}{\rho_{2}}\right) \text {. }
$$

The radii of normal curvature in the directions of the mean orthogonal lines are equal to the harmonic mean of the principal radii.

From (8) it follows that

The mean orthogonal lines on a minimal surface are asymptotic; and

The normal curvature of a surface of constant mean curvature in the direction of the mean orthogonal lines is constant all over the surface.

When in particular a mean orthogonal line on such a surface is a geodesic its first curvature is constant.

We have for any orthogonal system

$$
\frac{1}{\rho}+\frac{1}{\rho^{\prime}}=\frac{1}{\rho_{1}}+\frac{1}{\rho_{2}},
$$

$\rho, \rho^{\prime}$ denoting the normal radii in the given orthogonal directions. We assume that $\rho_{1}>\rho_{2}$; then since $\rho_{1}$ and $\rho_{2}$ are the limiting values we can put

$$
\frac{1}{\rho}=\frac{1}{\rho_{1}}+h, \quad \frac{1}{\rho^{\prime}}=\frac{1}{\rho_{2}}-h,
$$

where $h$ is an auxiliary function satisfying the conditions

Then

$$
h \nless 0, \quad h \ngtr \frac{1}{\rho_{2}}-\frac{1}{\rho_{1}} .
$$

$$
\frac{1}{\rho \rho^{\prime}}=\frac{1}{\rho_{1} \rho_{2}}+h\left(\frac{1}{\rho_{2}}-\frac{1}{\rho_{1}}-h\right) .
$$

From the above conditions it follows that the quantity in the parenthesis is always positive and consequently $1 / \rho \rho^{\prime}$ is minimum when $h$ is zero, and maximum when $h$ has the expression $\frac{1}{2}\left(1 / \rho_{2}-1 / \rho_{1}\right)$. Hence:

The product of the radii of normal curvature of a surface in orthogonal directions is least for the mean orthogonal lines and greatest for the lines of curvature.

From the Bertrand equation

$$
\frac{1}{\tau}=\frac{1}{2}\left(\frac{1}{\rho_{2}}-\frac{1}{\rho_{1}}\right) \sin 2 \theta
$$

where $\tau$ denotes the geodesic torsion of the curves whose tangent make an angle $\theta$ with the line of curvature corresponding to $\rho_{1}$, we find 


$$
\frac{1}{\tau_{u}}=-\frac{1}{\tau_{v}}=\frac{1}{2}\left(\frac{1}{\rho_{2}}-\frac{1}{\rho_{1}}\right) .
$$

From the form of (9) it is evident that this is the maximum value for $\tau$.* $^{*}$

KNOBLAUCH $\dagger$ shows that the distances $r_{u}, r_{v}$ have the following expressions:

From the relations

$$
\frac{1}{r_{u}}=\frac{e}{D}, \quad \frac{1}{r_{v}}=\frac{g}{D^{\prime \prime}} .
$$

$$
\frac{1}{\rho_{1} \rho_{2}}=\frac{D D^{\prime \prime}-D^{\prime 2}}{E G}, \quad \frac{1}{\rho_{1}}+\frac{1}{\rho_{2}}=-\frac{E D^{\prime \prime}+G D}{E G},
$$

one has in consequence of $(1)$

$$
\frac{1}{\rho_{1}}=-\frac{D}{E}+\frac{D^{\prime}}{\sqrt{E G}}, \quad \frac{1}{\rho_{2}}=-\frac{D}{E}-\frac{D^{\prime}}{\sqrt{E G}},
$$

From (11), (12), (13) and (c) we get

$$
\frac{1}{r_{u}}=\frac{1}{r_{v}}=\frac{G D^{2}+E D^{\prime 2}}{D E G}=\frac{\frac{1}{2}\left(\frac{1}{\rho_{1}^{2}}+\frac{1}{\rho_{2}^{2}}\right)}{\frac{1}{2}\left(\frac{1}{\rho_{1}}+\frac{1}{\rho_{2}}\right)} .
$$

If we note that the numerator of the last member is the expression for the Casorati curvature of the surface, we have the theorem:

The distance $r$ in the direction of a mean orthogonal line of a surface is equal to the ratio of the mean and Casorati curvatures of the surface at the point.

When the surface is mininal, $r$ is zero, hence the lines of striction of the ruled surfaces formed by the normals to a minimal surface along its asymptotic lines are the latter lines themselves.

Let $S$ be an isothermic surface, then if its lines of curvature are parametric, the equation of the mean orthogonal lines (2) can be brought to the form,

Consequently, if we put

$$
d u_{1}^{2}-d v_{1}^{2}=0 \text {. }
$$

$$
u_{1}-v_{1}=2 u, \quad u_{1}+v_{1}=2 v,
$$

$u$ and $v$ are the parameters of the mean orthogonal lines. Then the directions of the lines of curvature are given by the equation

$$
d u^{2}-d v^{2}=0
$$

* Bianchi, 1. c., p. 161.

† Einleitung in die Allgemenie Theorie der Krummen Flächen, p. 69. 
when the surface is referred to the mean orthogonal system. Comparing this with (5) we have $E=G$; in a similar manner it can be shown that when $E$ and $G$ are equal, $E_{1}$ and $G_{1}$ also are equal. Hence:

When a surface is isothermic, its mean orthogonal lines form an isothermal system and only in this case.

\section{$\S 2$. Lines conjugate to the mean orthogonal lines.}

The second system of lines is represented on the sphere by an orthogonal system, and when the surface is referred to this system, its coefficients satisfy the equations

$$
\frac{e}{E}=\frac{g}{G}, \quad \frac{e}{D}=\frac{g}{D^{\prime \prime}}, \quad f=0 .
$$

When the system of parametric lines on the surface is composed of the lines of curvature, the equation determining the directions of the system under consideration is

$$
e_{1} d u_{1}^{2}-g_{1} d v_{1}^{2}=0
$$

from which it is seen that these lines form equal angles with the lines of curvature. The above equation can be written

$$
G_{1} D_{1}^{2} d u_{1}^{2}-E_{1} D_{1}^{\prime \prime 2} d v_{1}^{2}=0,
$$

from which one finds that the directions of these lines make an angle $\theta$ with the directions of the lines of curvature $v=$ const. such that

$$
\tan \theta= \pm \frac{\rho_{2}}{\rho_{1}},
$$

where $\rho_{1}$ and $\rho_{2}$ are the principal radii. From this and (3) it follows that in the case of minimal surfaces and the sphere these lines coincide with the mean orthogonal lines; in the former case they are the asymptotic lines and in the latter any orthogonal system.

If $\theta$ and $\theta^{\prime}$ denote the angles which two conjugate directions upon a surface make with one of the lines of curvature, these functions satisfy the condition

$$
\tan \theta \tan \theta^{\prime}=-\frac{\rho_{2}}{\rho_{1}} .
$$

Comparing this result with (3) and (17), we note that the system of lines under consideration is composed of the lines conjugate to the lines of the mean orthogonal system. We shall refer to them as the conjugates of the mean orthogonal lines.

When this system of lines is parametric, equation (4) of the lines of curvature reduces to

$$
\left(E D^{\prime}-F D\right) d u^{2}-\left(G D^{\prime}-F D^{\prime \prime}\right) d v^{2}=0,
$$


which in consequence of $(15)$ reduces to

$$
e d u^{2}-g d v^{2}=0 \text {. }
$$

In a manner similar to that followed in the case of mean orthogonal lines we can establish the following theorem:

When the lines of curvature of a surface have an isothermal spherical representation, the representation of the conjugates of the mean orthogonal lines is isothermal and only in this case.

Given an orthogonal system on the sphere and put

$$
t=\frac{D}{e}=\frac{D^{\prime \prime}}{g}
$$

the Codazzi equations* can be written

$$
\begin{gathered}
g \frac{\partial t}{\partial u}-\frac{\partial D^{\prime}}{\partial v}+\frac{\partial}{\partial v} \log \sqrt{\frac{g}{e}} D^{\prime}=0, \\
e \frac{\partial t}{\partial v}-\frac{\partial D^{\prime}}{\partial u}+\frac{\partial}{\partial u} \log \sqrt{\frac{e}{g}} D^{\prime}=0 .
\end{gathered}
$$

The elimination of $t$ leads to the following equation which $D^{\prime}$ must satisfy:

$$
\begin{array}{r}
\frac{1}{e} \frac{\partial^{2} \phi}{\partial u}-\frac{1}{g} \frac{\partial^{2} \phi}{\partial v^{2}}+\frac{1}{e}\left(\frac{\partial \log \sqrt{g}}{\partial u}-3 \frac{\partial \log \sqrt{e}}{\partial u}\right) \frac{\partial \phi}{\partial u} \\
(20)-\frac{1}{g}\left(\frac{\partial \log \sqrt{e}}{\partial v}-3 \frac{\partial \log \sqrt{g}}{\partial v}\right) \frac{\partial \phi}{\partial v}+\left[\frac{1}{e}\left(\frac{\partial^{2}}{\partial u^{2}} \log \sqrt{\frac{g}{e}}-\frac{\partial}{\partial u} \log \sqrt{\frac{g}{e}} \frac{\partial \log e}{\partial u}\right)\right. \\
\left.\quad-\frac{1}{g}\left(\frac{\partial^{2}}{\partial v^{2}} \log \sqrt{\frac{e}{g}}-\frac{\partial}{\partial v} \log \sqrt{\frac{e}{g}} \frac{\partial \log g}{\partial v}\right)\right] \phi=0 .
\end{array}
$$

From this it follows that any orthogonal system on the sphere is the representation of the conjugates of the mean orthogonal lines on certain surfaces. For when a particular integral $D^{\prime}$ is known of the corresponding equation (20), one gets $t$ from (19) by a quadrature and then $D$ and $D^{\prime \prime}$ from (18). The cartesian coördinates of the surface are given by quadratures from

$$
\frac{\partial x}{\partial u}=-\frac{D}{e} \frac{\partial X}{\partial u}-\frac{D^{\prime}}{g} \frac{\partial X}{\partial v}, \quad \frac{\partial x}{\partial v}=-\frac{D^{\prime}}{e} \frac{\partial X}{\partial u}-\frac{D^{\prime \prime}}{g} \frac{\partial X}{\partial v},
$$

and similarly for $y$ and $z$, where $X, Y, Z$ are the coördinates of the sphere.

When the system upon the sphere is isothermal and the parameters are chosen so that

$$
e=g=\lambda
$$

* BiANChi, 1. c., p. 120. 
the equation $(20)$ reduces to

$$
\frac{\partial}{\partial u}\left(\frac{1}{\lambda} \frac{\partial \phi}{\partial u}\right)=\frac{\partial}{\partial v}\left(\frac{1}{\lambda} \frac{\partial \phi}{\partial v}\right) .
$$

If we denote by $\alpha$ and $\beta$ the parameters of the lines of curvature of a surface with this representation of these lines, we have from the preceding discussion

$$
\alpha=u+v, \quad \beta=u-v,
$$

so that the above equation becomes by change of variables

$$
\frac{\partial^{2} \phi}{\partial \alpha \partial \beta}-\frac{\partial \log \sqrt{\lambda} \partial \phi}{\partial \beta}-\frac{\partial \log \sqrt{\lambda} \partial \phi}{\partial \alpha}=0 .
$$

Hence the problem of finding surfaces with an isothermai spherical representation of the conjugates of the mean orthogonal lines, or, what is the same thing, such a representation of the lines of curvature reduces to the integration of an equation of Laplace with equal invariants.

In closing this discussion of the conjugates of the mean orthogonal lines upon a surface, we remark from (15) that the normals to the surface along these lines form the mean ruled surfaces of the congruence of normals.*

\section{§3. Characteristic lines.}

This system of lines is conjugate in such a way that when it is parametric its functions satisfy the conditions

$$
\frac{D}{E}=\frac{D^{\prime \prime}}{G}, \quad \frac{D}{e}=\frac{D^{\prime \prime}}{g}, \quad D^{\prime}=0 .
$$

As we shall consider only real lines it is assumed that $S^{r}$ has positive total curvature. The general equation of the directions of this system is readily found to be

$$
\begin{aligned}
& {\left[D\left(G D-E D^{\prime \prime}\right)-2 D^{\prime}\left(F D-E D^{\prime}\right)\right] d u^{2}} \\
& \quad+\left[2 D^{\prime}\left(G D+E D^{\prime \prime}\right)-4 F D D^{\prime \prime}\right] d u d v \\
& \quad+\left[2 D^{\prime}\left(G D^{\prime}-F D^{\prime \prime}\right)-D^{\prime \prime}\left(G D-E D^{\prime \prime}\right)\right] d v^{2}=0
\end{aligned}
$$

When the lines of curvature are parametric, the equation for the determination of these lines is

$$
D_{1} d u_{1}^{2}-D_{1}^{\prime \prime} d v_{1}^{2}=0
$$

And from the general equation (4) of the lines of curvature, it follows that

* Cif a Relli, l. c. 
when the surface is referred to this conjugate system, the directions of the lines of curvature are given by

$$
D d u^{2}-D^{\prime \prime} d v^{2}=0 .
$$

From equations $\left(24^{\prime}\right)$ and (25) we get the theorem:

The necessary and sufficient condition that the characteristic lines upon a surface form an isothermal-conjugate system is that the lines of curvature are isothermal-conjugate.

From $\left(24^{\prime}\right)$ it is seen that the angle between the characteristic directions is bisected by the lines of curvature. Moreover, this is the only conjugate system whose included angle is thus bisected. For, the directions bisecting the angles between any parametric system on a surface are given by

$$
E d u^{2}-G d v^{2}=0 .
$$

When the surface is referred to any conjugate system the equation (4) of the lines of curvature becomes

$$
F D d u^{2}+\left(G D-E D^{\prime \prime}\right) d u d v-F D^{\prime \prime} d v^{2}=0 ;
$$

and the only way in which this equation can take the above form is given by

$$
G D-E D^{\prime \prime}=0,
$$

which characterizes the conjugate system under discussion. Consequently this conjugate system on surfaces of positive curvature is an analogue of the asymptotic system on surfaces of negative curvature.

Denoting by $\phi$ the angle which the characteristic directions form with the tangent to the line of curvature of normal radius $\rho_{1}$, we have from $\left(24^{\prime}\right)$

$$
\tan \phi= \pm \sqrt{\frac{G}{E}} \frac{d v}{d u}= \pm \sqrt{\frac{\rho_{2}}{\rho_{1}}} *
$$

Comparing (3), (17) and (29) and assuming that $\rho_{1}$ is greater than $\rho_{2}$, we see that the mean orthogonal lines make angles $\pi / 4$ with the tangent to the line of curvature of normal radius $\rho_{1}$, the characteristic directions make equal and less angles, and the conjugates of the first make angles which are still less and equal to one another.

By following the method employed in the study of the mean orthogonal lines, one finds readily the expressions for the normal curvature, the geodesic torsion and the distance $r$ for the directions of the second system of lines and the characteristic directions in terms of the principal radii. We give the results for all three systems in the following table :

* If we denote by $\theta$ the similar angle for asymptotic lines on a surface of negative curvature, we find $\tan \theta= \pm \sqrt{-\rho_{2} / \rho_{1}}$. 


$$
\begin{array}{l|c|c|c}
\frac{1}{\rho_{u}}=\frac{1}{\rho_{v}}= & \mathrm{II} & \mathrm{III} \\
\frac{1}{\tau_{u}}=-\frac{1}{\tau_{v}}= & \frac{1}{2}\left(\frac{1}{\rho_{1}}+\frac{1}{\rho_{2}}\right) & \frac{\rho_{1}+\rho_{2}}{\rho_{1}^{2}+\rho_{2}^{2}} & \frac{2}{\rho_{1}+\rho_{2}} \\
\frac{1}{2}\left(\frac{1}{\rho_{2}}-\frac{1}{\rho_{1}}\right) & \frac{\rho_{1}-\rho_{2}}{\rho_{1}^{2}+\rho_{2}^{2}} & \sqrt{\rho_{1} \rho_{2}} \frac{\rho_{1}-\rho_{2}}{\rho_{1}+\rho_{2}} \\
\frac{1}{r_{u}}=\frac{1}{r_{v}}= & \frac{\left(\rho_{1}^{2}+\rho_{2}^{2}\right)}{\rho_{1} \rho_{2}\left(\rho_{1}+\rho_{2}\right)} & \frac{2}{\rho_{1}+\rho_{2}} & \frac{1}{2}\left(\frac{1}{\rho_{1}}+\frac{1}{\rho_{2}}\right)
\end{array}
$$

Between the normal radii of any two conjugate directions the following relation obtains, *

$$
\rho+\rho^{\prime}=\rho_{1}+\rho_{2} \text {. }
$$

By considerations similar to those used in the case of mean orthogonal lines we deduce from this relation the theorem:

The product of the radii of curvature in conjugate directions is maximum when the directions are characteristic and minimum for the lines of curvature.

From the value of $r$ for characteristic lines, we have the theorem:

In a ruled surface formed by the normals to a surface of constant mean curvature with positive total curvature along a characteristic line the line of striction is at a constant distance from the surface.

Let $S$ be a surface of constant mean curvature $1 / 2 a$, then the lines of striction of all the ruled surfaces above considered lie on the surface $S_{0}$ defined by $\dagger$

$$
x_{0}=x+2 a X, \quad y_{0}=y+2 a Y, \quad z_{0}=z+2 a Z,
$$

where $X, Y, Z$ are the direction-cosines of the normal to $S$. Since $a$ is constant, $S_{0}$ is parallel to $S$.

Between the radii of principal curvature of $S$ and $S_{0}$ the following relations hold : $\ddagger$

$$
\rho_{1}^{0}=\rho_{1}-2 a, \quad \rho_{2}^{0}=\rho_{2}-2 a,
$$

from which one finds that $S_{0}$ has mean curvature $-1 / 2 a$. From the general relations

$$
\begin{aligned}
& D_{0}=2 E \frac{a}{\rho_{1} \rho_{2}}+D\left[1-2 a\left(\frac{1}{\rho_{1}}+\frac{1}{\rho_{2}}\right)\right], \\
& D_{0}^{\prime \prime}=2 \frac{G a}{\rho_{1} \rho_{2}}+D^{\prime \prime}\left[1-2 a\left(\frac{1}{\rho_{1}}+\frac{1}{\rho_{2}}\right)\right],
\end{aligned}
$$

it results by means of (30) that $D_{0}$ and $D_{0}^{\prime \prime}$ are zero, so that the asymptotic lines on $S_{0}$ correspond to the characteristic lines on $S$. We have then the theorem:

* KNobla UCh, l. c., p. 56.

† KNOBLAUCH, 1. c., p. 69.

$\ddagger$ Ibid., p. 236. 
The ruled surfaces formed by the normals to a surface, of positive curvature and with mean curvature $1 / 2 a$, along the characteristic lines cut the parallel surface of mean curvature $-1 / 2 a$ in its asymptotic lines and have the latter for their lines of striction.

From the equation (29) it is seen that on the Weingarten surfaces whose radii are bound by a relation of the form

$$
\frac{\rho_{1}}{\rho_{2}}=\text { constant }
$$

the characteristic lines cut at constant angle and only in this case.* Moreover, the conjugates of the mean orthogonal lines also cut under constant angle, so that the configuration of all three systems is the same at all points of such a surface.

One of the characteristic properties of Weingarten surfaces is that the asymptotic lines on the sheets of the evolute correspond. We inquire whether there are any surfaces for which the characteristic lines on the sheets of the evolute correspond. Let the focal nappes be referred to the conjugate system corresponding to the lines of curvature of the surface; then the direction of the characteristic lines on the first nappe are given by the equation

$$
D_{1}\left(G_{1} D_{1}-E_{1} D_{1}^{\prime \prime}\right) d u^{2}-4 F_{1}^{\prime} D_{1} D_{1}^{\prime \prime} d u d v+D_{1}^{\prime \prime}\left(E_{1} D_{1}^{\prime \prime}-G_{1} D_{1}\right) d v^{2}=0,
$$

where the subscripts denote that the functions belong to the first nappe; and the directions of the lines of curvature are given by

$$
F_{1} D_{1} d u^{2}+\left(G_{1} D_{1}-E_{1} D_{1}^{\prime \prime}\right) d u d v-F_{1} D_{1}^{\prime \prime} d v^{2}=0 \text {. }
$$

To get the equations for the second nappe we have only to change the subscript. Now the necessary and sufficient condition that the characteristic lines correspond on the two nappes is

$$
\frac{D_{1}\left(G_{1} D_{1}-E_{1} D_{1}^{\prime \prime}\right)}{D_{2}\left(G_{2} D_{2}-E_{2} D_{2}^{\prime \prime}\right)}=\frac{F_{1} D_{1} D_{1}^{\prime \prime}}{F_{2} D_{2} D_{2}^{\prime \prime}}=\frac{D_{1}^{\prime \prime}\left(E_{1} D_{1}^{\prime \prime}-G_{1} D_{1}\right)}{D_{2}^{\prime \prime}\left(E_{2} D_{2}^{\prime \prime}-G_{2} D_{2}\right)},
$$

which reduces to

$$
\frac{G_{1}^{\prime} D_{1}-E_{1} D_{1}^{\prime \prime}}{G_{2} D_{2}-E_{2}^{\prime} D_{2}^{\prime \prime}}=\frac{F_{1} D_{1}}{F_{2} D_{2}}=\frac{F_{1} D_{1}^{\prime \prime}}{F_{2} D_{2}^{\prime \prime}}
$$

From the last two we have that the asymptotic lines correspond on the two sheets and consequentiy $S$ is a Weingarten surface. Moreover, from the above equation of the lines of curvature it follows that the lines of curvature on the two nappes correspond and conversely when this condition is satisfied the above rela-

\footnotetext{
* It is evident that on the surfaces whose radii satisfy (31) any function of the tangent of the angle between the characteristic lines is constant. Massimi (Atti dell'Accad. Pont. dei Nuovi Lincei, vol. 52, p. 130) has shown that this is true for every conjugate system of lines on these Weingarten surfaces and only for these.
} 
tions hold. But the only $W$ surfaces whose focal sheets have corresponding lines of curvature are those corresponding to a relation of the form

$$
\rho_{1}-\rho_{2}=\text { constant. } *
$$

But the focal sheets in this case are pseudo-spherical surfaces and consequently the characteristic lines are imaginary.

In the preceding discussion we have not treated the case where the conjugate lines upon the sheets corresponding to the lines of curvature on the surface are the characteristic lines. Making use of the expression given by KNOBLAUCH $\dagger$ for the fundamental coefficients of the two nappes in terms of the functions for $S$ referred to its lines of curvature, we have the following conditions to be satisfied

$$
\begin{aligned}
& {\left[G \rho_{1}^{2} \frac{\partial \rho_{1}}{\partial u} \frac{\partial \rho_{2}}{\partial u}-E \rho_{2}^{2}\left(\frac{\partial \rho_{1}}{\partial v}\right)^{2}-E G\left(\rho_{1}-\rho_{2}\right)^{2}\right] \frac{\partial \rho_{1}}{\partial u}=0,} \\
& {\left[E \rho_{2}^{2} \frac{\partial \rho_{1}}{\partial v} \frac{\partial \rho_{2}}{\partial v}-G \rho_{1}^{2}\left(\frac{\partial \rho_{2}}{\partial u}\right)^{2}-E G\left(\rho_{1}-\rho_{2}\right)^{2}\right] \frac{\partial \rho_{2}}{\partial v}=0 .}
\end{aligned}
$$

If $\rho_{1}$ is a function of $v$ alone, or $\rho_{2}$ of $u$ alone, one of the focal nappes reduces to a curve, so that this case cannot arise. Consequently the parentheses must be zero, but these are proportional $\dagger$ to the mean curvatures of the focal sheets, so that the latter must be minimal surfaces and therefore surfaces of negative curvature. Hence the theorem:

There are no surfaces whose focal sheets are of positive total curvature and have their characteristic lines in correspondence.

\section{§4. Spherical representalion of characteristic lines.}

Consider a surface referred to its real characteristic lines. The following relations hold between the differential quotients of its rectangular coördinates and of the direction-cosines of the normal $\ddagger$

$$
\begin{aligned}
& \frac{\partial x}{\partial u}=-\frac{D}{e g-f^{2}}\left(g \frac{\partial X}{\partial u}-f \frac{\partial X}{\partial v}\right), \\
& \frac{\partial x}{\partial v}=\frac{D^{\prime \prime}}{e g-f^{2}}\left(f^{\frac{\partial X}{\partial u}}-e \frac{\partial X}{\partial v}\right),
\end{aligned}
$$

with analogous expressions in $y$ and $z$. If we put

$$
\frac{D \sqrt{g}}{\sqrt{e} \cdot \sqrt{e g-f^{2}}}=\frac{D^{\prime \prime} \sqrt{e}}{\sqrt{g} \cdot \sqrt{e g-f^{2}}}=\lambda,
$$

\footnotetext{
* BiANChi, l. c., p. 234.

† L. c., p. 218.

† BIANCHI, 1. c., p. 119.
}

Trans. Am. Math. Soc. 29 
the second of conditions (23) is satisfied and

$$
\lambda^{2}=\frac{1}{K} .
$$

When the substitution is made in the above, they become

$$
\begin{aligned}
& \frac{\partial x}{\partial u}=-\lambda \sqrt{\frac{e}{g}}\left(\frac{g}{\sqrt{e g-f^{2}}} \frac{\partial X}{\partial u}-\frac{f}{\sqrt{e g-f^{2}}} \frac{\partial X}{\partial v}\right), \\
& \frac{\partial x}{\partial v}=\lambda \sqrt{\frac{g}{e}}\left(\frac{f}{\sqrt{e g-f^{2}}} \frac{\partial X}{\partial u}-\frac{e}{\sqrt{e g-f^{2}}} \frac{\partial X}{\partial v}\right),
\end{aligned}
$$

which by means of a set of formulæ given by BIANCHI* can be brought to the form

$$
\frac{\partial x}{\partial u}=\lambda \sqrt{\frac{e}{g}}\left|\begin{array}{cc}
Y & Z \\
\frac{\partial Y}{\partial v} & \frac{\partial Z}{\partial v}
\end{array}\right|, \quad \frac{\partial x}{\partial v}=-\lambda \sqrt{\frac{g}{e}}\left|\begin{array}{cc}
Y & Z \\
\frac{\partial Y}{\partial u} & \frac{\partial Z}{\partial u}
\end{array}\right| .
$$

By means of (32) the Codazzi equations can be written

$$
\begin{aligned}
& \frac{\partial \log \lambda}{\partial u}=\frac{\partial}{\partial u} \log \sqrt{\frac{e}{g}}-\left\{\begin{array}{c}
22 \\
1
\end{array}\right\}^{\prime} \frac{e}{g}-\left\{\begin{array}{c}
11 \\
1
\end{array}\right\}^{\prime}, \\
& \frac{\partial \log \lambda}{\partial v}=\frac{\partial}{\partial v} \log \sqrt{\frac{g}{e}}-\left\{\begin{array}{c}
11 \\
2
\end{array}\right\}^{\prime} \frac{g}{e}-\left\{\begin{array}{c}
22 \\
2
\end{array}\right\}^{\prime},
\end{aligned}
$$

where the Christoffel symbols $\left\{\begin{array}{c}r_{s} \\ t\end{array}\right\}^{\prime}$ are formed with respect to the linear element of the spherical representation. Expressing the condition of integrability of the above equations, we are brought to the theorem :

The necessary and sufficient condition that a system of lines upon the sphere shall be the spherical representation of the characteristic lines upon a surface is that the following equation be satisfied:

$$
\frac{\partial}{\partial u}\left[\left\{\begin{array}{c}
11 \\
2
\end{array}\right\}^{\prime} \frac{g}{e}+\left\{\begin{array}{c}
22 \\
2
\end{array}\right\}^{\prime}+\frac{\partial}{\partial v} \log \sqrt{\frac{e}{g}}\right]=\frac{\partial}{\partial v}\left[\left\{\begin{array}{c}
22 \\
1
\end{array}\right\}^{\prime} \frac{e}{g}+\left\{\begin{array}{c}
11 \\
1
\end{array}\right\}^{\prime}+\frac{\partial}{\partial u} \sqrt{\frac{g}{e}}\right] \cdot \dagger
$$

Where this condition is satisfied and the coördinates of the sphere have been found, the complete determination of the surface is given by the quadratures (35) and (34).

When a surface is referred to any conjugate system, one has $\ddagger$

$$
E=\frac{g D^{2}}{e g-f^{2}}, \quad F=-\frac{f D D^{\prime \prime}}{e g-f^{2}}, \quad G=\frac{e D^{\prime 2}}{e g-f^{2}} .
$$

* L. c., p. 128.

†This equation of condition bas been found by RAFFY, l. c., p. 232.

$\ddagger$ BIANCHI, l. c., p. 117. 
When the system is composed of the characteristic lines, these relations can, by means of (32), be put in the form

$$
E=\lambda^{2} e, \quad F=-\lambda^{2} f, \quad G=\lambda^{2} g .
$$

Conversely, in order that equations (37) can take this form, it is necessary that

or

$$
\frac{g D^{2}}{e}=D D^{\prime \prime}=\frac{e D^{\prime 2}}{g},
$$

Hence the theorem :

$$
\frac{D}{e}=\frac{D^{\prime \prime}}{g}
$$

The necessary and sufficient condition that the linear element of a surface of positive curvature, referred to a conjugate system, can be written in the form

$$
d s^{2}=\lambda^{2}\left(e d u^{2}-2 f d u d v+g d v^{2}\right)
$$

is that the conjugate system be composed of the characteristic lines.

Here again the analogy between characteristic lines and asymptotic lines is apparent.

If we put

$$
\xi=\sqrt{\lambda} X, \quad \eta=\sqrt{\lambda} Y, \quad \zeta=\sqrt{\lambda} Z,
$$

the equations (34) reduce to

$$
\frac{\partial x}{\partial u}=\sqrt{\frac{e}{g}}\left|\begin{array}{cc}
\eta & \zeta \\
\frac{\partial \eta}{\partial v} & \frac{\partial \zeta}{\partial v}
\end{array}\right|, \quad \frac{\partial x}{\partial v}=\sqrt{\frac{g}{e}}\left|\begin{array}{cc}
\eta & \zeta \\
\frac{\partial \eta}{\partial u} & \frac{\partial \zeta}{\partial u}
\end{array}\right|,
$$

and similar expressions in $y$ and $z$. From the following two equations which $X, Y, Z$ satisfy*

$$
\begin{aligned}
& \frac{\partial^{2} \phi}{\partial u^{2}}-\left\{\begin{array}{c}
11 \\
1
\end{array}\right\}^{\prime} \frac{\partial \phi}{\partial u}-\left\{\begin{array}{c}
11 \\
2
\end{array}\right\}^{\prime} \frac{\partial \phi}{\partial v}+e \phi=0, \\
& \frac{\partial^{2} \phi}{\partial v^{2}}-\left\{\begin{array}{c}
22 \\
1
\end{array}\right\}^{\prime} \frac{\partial \phi}{\partial u}-\left\{\begin{array}{c}
22 \\
2
\end{array}\right\}^{\prime} \frac{\partial \phi}{\partial v}+g \phi=0,
\end{aligned}
$$

we find by aid of (35) that they are also solutions of

$$
\begin{aligned}
\sqrt{\frac{g}{e}} \frac{\partial^{2} \phi}{\partial u}+\sqrt{\frac{e}{g}} \frac{\partial^{2} \phi}{\partial v^{2}}+\sqrt{\frac{g}{e}} & {\left[\frac{\partial \log \lambda}{\partial u}+\frac{\partial}{\partial u} \log \sqrt{\frac{g}{e}}\right] \frac{\partial \phi}{\partial u} } \\
& +\sqrt{\frac{e}{g}}\left[\frac{\partial \log \lambda}{\partial v}+\frac{\partial}{\partial v} \log \sqrt{\frac{e}{g}}\right] \frac{\partial \phi}{\partial v}+2 \sqrt{e g} \phi=0 .
\end{aligned}
$$

* Bianchi, l. c., p. 119. 
If now we put

the above equation reduces to

$$
\sqrt{\lambda} \phi=\theta
$$

$$
\begin{gathered}
\frac{\partial}{\partial u}\left(\sqrt{\frac{g}{e}} \frac{\partial \theta}{\partial u}\right)+\frac{\partial}{\partial v}\left(\sqrt{\frac{e}{g}} \frac{\partial \theta}{\partial v}\right)=M \theta \\
M \equiv \frac{1}{\sqrt{\lambda}}\left[\frac{\partial}{\partial u}\left(\sqrt{\frac{g}{e}} \frac{\partial \sqrt{\lambda}}{\partial u}\right)+\frac{\partial}{\partial v}\left(\sqrt{\frac{e}{g}} \frac{\partial \sqrt{\lambda}}{\partial v}\right)\right]-2 \sqrt{e g} .
\end{gathered}
$$

It is seen that this equation is satisfied by $\xi, \eta, \zeta$.

Conversely, given any equation of the form

$$
\frac{\partial}{\partial u}\left(\frac{1}{\mu} \frac{\partial \theta}{\partial u}\right)+\frac{\partial}{\partial v}\left(\mu \frac{\partial \theta}{\partial v}\right)=M \theta
$$

where $\mu$ and $M$ are any functions of $u$ and $v$, the surface whose coördinates are obtained by quadratures from

$$
x=\int \mu\left|\begin{array}{cc}
\eta & \zeta \\
\frac{\partial \eta}{\partial v} & \frac{\partial \zeta}{\partial v}
\end{array}\right| d u-\frac{1}{\mu}\left|\begin{array}{cc}
\eta & \zeta \\
\frac{\partial \eta}{\partial u} & \frac{\partial \zeta}{\partial u}
\end{array}\right| d v,
$$

and similarly for $y$ and $z$, where $\xi, \eta, \zeta$ are solutions of the above equation, has the curves $u=$ const., $v=$ const., for a conjugate system. Moreover, if the corresponding functions $e$ and $g$ satisfy the condition

$$
\frac{e}{g}=\mu^{2},
$$

these curves are the characteristic lines. From (39) it follows that a necessary condition that these lines be characteristic on the above surface is

$$
\frac{E}{\bar{G}}=\mu^{2},
$$

but from (37) it is evident that this is not a sufficient condition.

We have seen that the characteristic lines from an isothermal conjugate system only on surfaces with isothermal conjugate lines of curvature. In this case by a suitable choice of the parameters of the characteristic lines we have $D$ and $D^{\prime \prime}$ equal, so that $e$ and $g$ are equal. When $e$ and $g$ are made equal in the preceding equations, they take the forms given by BIANCHI to the equations of surfaces referred to any isothermal conjugate system.

In closing we call attention to the fact that the differential equations of the three systems of lines which have been considered reduces, when the surface is referred to its lines of curvature, to the form

$$
\psi^{2} \equiv \lambda d u^{2}-\mu d v^{2}=0,
$$


when $\lambda, \mu$ have the respective values $E, G ; e, g ; D, D^{\prime \prime}$ according as this equation defines curves of the first, second or third class. For this parametric system the forms $d s^{2}, \phi^{2}$ and $d \sigma^{2}$ lack the middle term. The Jacobian of $\psi^{2}$ and any of the last three is of the form

$$
\chi^{2} \equiv t d u d v,
$$

where $t$ vanishes in case $S$ is a minimal surface, which class of surfaces must be excluded from the consideration as we have seen before. If in accordance with the Crfarelli theorem we form the Jacobian of $\chi^{2}$ and $d s^{2}, \phi^{2}$ or $d \sigma^{2}$, we are brought to one of the forms $\psi^{2}$. The only one of the forms $\psi^{2}$ which is definite is

$$
D d u^{2}-D^{\prime \prime} d v^{2} \text {, }
$$

for surfaces of negative curvature. The Jacobian of this and $\chi^{2}$ gives $\phi^{2}$, hence we are brought to asymptotic lines. Since the latter may be considered as forming a system of lines from which we started, we may say that the repeated application of this theorem of CIFARELLI does not give rise to any lines other than those of the three systems considered.

Princeton University, January, 1904. 